\title{
SECONDARY THEORIES FOR SIMPLICIAL MANIFOLDS AND CLASSIFYING SPACES
}

\author{
MARCELLO FELISATTI AND FRANK NEUMANN
}

\begin{abstract}
We define secondary theories and characteristic classes for simplicial smooth manifolds generalizing Karoubi's multiplicative Ktheory and multiplicative cohomology groups for smooth manifolds. As a special case we get versions of the groups of differential characters of Cheeger and Simons for simplicial smooth manifolds. Special examples include classifying spaces of Lie groups and Lie groupoids.
\end{abstract}

\section{INTRODUCTION}

We introduce and analyze secondary theories and characteristic classes for bundles with connections on simplicial smooth manifolds.

Classical Cheeger-Simons differential characters for simplicial smooth manifolds with respect to Deligne's 'filtration bête' [De] of the associated de Rham complex were first introduced by Dupont-Hain-Zucker [DHZ] in order to study the relation between the Cheeger-Chern-Simons invariants of vector bundles with connections on smooth algebraic varieties and the corresponding characteristic classes in Deligne-Beilinson cohomology.

In the case of a smooth manifold Dupont, Hain and Zucker showed that the group of Cheeger-Simons differential characters is isomorphic to the cohomology group of the cone of the natural map from Deligne's 'filtration bête' on the de Rham complex of the manifold to the complex of smooth singular cochains.

In a series of fundamental papers Karoubi [K1], [K2] introduced multiplicative K-theory and multiplicative cohomology groups, defined for any filtration of the de Rham complex of a smooth manifold. By taking the filtration to be the 'filtration bête' it follows that Karoubi's multiplicative cohomology groups are generalizations of the classical Cheeger-Simons differential characters in appropriate degrees.

The first author in $[\mathrm{F}]$ studied the relationship between differential characters and multiplicative cohomology further. He gave a definition of differential characters associated to an arbitrary filtration of the de Rham complex, which in the case of the 'filtration bête' reduces again to the classical case

1991 Mathematics Subject Classification. Primary 57R20, 55R40.

Key words and phrases. multiplicative K-theory, multiplicative cohomology, differential characters, smooth simplicial manifolds, simplicial de Rham theory. 
of Cheeger-Simons. The advantage is that this more general definition allows for the definition of an explicit map at the levels of cocycles between Karoubi's multiplicative cohomology groups and Cheeger-Simons differential characters. It turns out that Karoubi's multiplicative cohomology groups are the natural gadgets for systematically constructing and studying secondary characteristic classes.

Following a similiar route in this article we generalize Karoubi's multiplicative cohomology groups and the groups of Cheeger-Simons differential characters even further to simplicial smooth manifolds and arbitrary filtrations of the associated simplicial de Rham complex and study their relations. This allows for a wider range of applications, for example to classifying spaces of Lie groups and Lie groupoids.

The outline of the paper is as follows: After introducing the main background of simplicial de Rham and Chern-Weil theory, mainly following Dupont [D1], [D2] we introduce multiplicative cohomology groups and groups of differential characters for arbitrary filtrations of the simplicial de Rham complex. We discuss briefly some examples like classifying spaces of Lie groups and Lie groupoids. After introducing the concept of multiplicative bundles and multiplicative K-theory on smoooth simplicial manifolds, we construct characteristic classes of elements in the multiplicative K-theory with values in multiplicative cohomology and in the groups of differential characters.

In a sequel to this paper we will use this approach to construct and study in a unifying way secondary theories and characteristic classes for smooth manifolds, foliations, orbifolds, differentiable stacks etc. basically for everything to which one can associate a groupoid whose nerve gives rise to a simplicial smooth manifold. Differential characters for orbifolds were already introduced by Lupercio and Uribe using closely the approach of Hopkins and Singer [HS]. Chern-Weil theory for general etale groupoids was systematically analyzed by Crainic and Moerdijk $[\mathrm{CM}]$ using a very elegant approach to Cech-de Rham theory, which especially applies well to leaf spaces of foliated manifolds. Working instead in the algebraic geometrical context using de Rham theory for simplicial schemes a similiar machinery allows for defining secondary characteristic classes for Deligne-Mumford stacks, most prominently for the moduli stack of families of algebraic curves. Especially multiplicative cohomology with respect to the Hodge or Hodge-Deligne filtration will be of special interest here. Algebraic Cheeger-Simons differential characters for algebraic bundles with connections on smooth algebric varieties were already studied systematically by Esnault [E1], [E2].

Acknowledgements. The first author was supported by EPSRC research grant GR/S08046/01. The second author likes to thank the organizers of the Hanoi Conference in Algebraic Topology for organizing this wonderful conference and for partial financial support. Final parts of the work were completed while he was visiting the Tata Institute of Fundamental Research 
in Mumbai and he likes to thank the algebraic geometry group and especially N. Nitsure for a very inspiring stay. Both authors also like to thank J. R. Hunton for many useful discussions.

\section{Elements of simplicial de Rham and Chern-Weil theory}

We recall the ingredients of simplicial de Rham and Chern-Weil theory as can be found in [D1], [D2] or [DHZ].

A simplicial smooth manifold $X_{\bullet}$ is a simplicial object in the category of $\mathcal{C}^{\infty}$-manifolds. In other words a simplicial smooth manifold is a functor

$$
X_{\bullet}: \Delta^{o p} \rightarrow\left(\mathcal{C}^{\infty}-\text { manifolds }\right)
$$

We can think of $X_{\bullet}$ as a collection $X_{\bullet}=\left\{X_{n}\right\}$ of smooth manifolds $X_{n}$ for $n \geq 0$ together with smooth face and degeneracy maps

$$
\varepsilon_{i}: X_{n} \rightarrow X_{n-1}, \eta_{i}: X_{n} \rightarrow X_{n+1}
$$

for $0 \leq i \leq n$ such that the usual simplicial identities hold. These maps are functorially associated to the inclusion and projection maps

$$
\varepsilon^{i}: \Delta^{n-1} \rightarrow \Delta^{n}, \eta^{i}: \Delta^{n+1} \rightarrow \Delta^{n} .
$$

For the differential geometric constructions on $X_{\bullet}$ as introduced below, the degeneracy maps play no role and everything can be defined for so-called strict simplicial or $\Delta$-manifolds [DHZ].

The fat realization of a simplicial space $X \bullet$ is the quotient space

$$
\|X \bullet\|=\coprod_{n \geq 0}\left(\Delta^{n} \times X_{n}\right) / \sim
$$

where the equivalence relation is generated by

$$
\left(\varepsilon^{i} \times i d\right)(t, x) \sim\left(i d \times \varepsilon_{i}\right)(t, x)
$$

for any $(t, x) \in \Delta^{n-1} \times X_{n}$.

There are two versions of the de Rham complex on a simplicial manifold $X \bullet$ [D1], [D2].

The de Rham complex of compatible forms. A simplicial smooth complex $k$-form $\omega$ on $X_{\bullet}$ is a sequence $\left\{\omega^{(n)}\right\}$ of smooth complex $k$-forms $\omega^{(n)} \in \Omega_{d R}^{k}\left(\Delta^{n} \times X_{n}\right)$ satisfying the compatibility condition

$$
\left(\varepsilon^{i} \times i d\right)^{*} \omega^{(n)}=\left(i d \times \varepsilon_{i}\right)^{*} \omega^{(n-1)}
$$

in $\Omega_{d R}^{k}\left(\Delta^{n-1} \times X_{n}\right)$ for all $0 \leq i \leq n$ and all $n \geq 1$. Let $\Omega_{d R}^{k}\left(X_{\bullet}\right)$ be the set of all simplicial smooth complex $k$-forms on $X_{\bullet}$. The exterior differential on $\Omega_{d R}^{k}\left(\Delta^{n} \times X_{n}\right)$ induces an exterior differential d on $\Omega_{d R}^{k}\left(X_{\bullet}\right)$. We denote by $\left(\Omega_{d R}^{*}\left(X_{\bullet}\right), \mathrm{d}\right)$ the de Rham complex of compatible forms.

We note that $\left(\Omega_{d R}^{*}\left(X_{\bullet}\right), \mathrm{d}\right)$ is the total complex of a double complex $\left(\Omega_{d R}^{*, *}\left(X_{\bullet}\right), \mathrm{d}^{\prime}, \mathrm{d}^{\prime \prime}\right)$ with

$$
\Omega_{d R}^{k}\left(X_{\bullet}\right)=\bigoplus_{r+s=k} \Omega_{d R}^{r, s}\left(X_{\bullet}\right)
$$


and $\mathrm{d}=\mathrm{d}^{\prime}+\mathrm{d}^{\prime \prime}$, where $\Omega_{d R}^{r, s}\left(X_{\bullet}\right)$ is the vector space of $(r+s)$-forms, which when restricted to $\Delta^{n} \times X_{n}$ are locally of the form

$$
\left.\omega\right|_{\Delta^{n} \times X_{n}}=\sum a_{i_{1} \ldots i_{r} j_{1} \ldots j_{s}} \mathrm{~d} t_{i_{1}} \wedge \ldots \mathrm{d} t_{i_{r}} \wedge \mathrm{d} x_{j_{1}} \wedge \mathrm{d} x_{j_{s}},
$$

where $\left(t_{0}, \ldots, t_{n}\right)$ are barycentric coordinates of $\Delta^{n}$ and the $\left\{x_{j}\right\}$ are local coordinates of $X_{n}$. Furthermore the differentials $\mathrm{d}^{\prime}$ and $\mathrm{d}^{\prime \prime}$ are the exterior differentials on $\Delta^{n}$ and $X_{n}$ respectively.

We remark that $\omega=\left\{\omega^{(n)}\right\}$ defines a smooth $k$-form on

$$
\coprod_{n \geq 0}\left(\Delta^{n} \times X_{n}\right)
$$

and the compatible condition is the necessary and sufficient condition to define a form on the fat realization $\left\|X_{\bullet}\right\|$ of $X_{\bullet}$ in view of the generating equivalence relation for defining the quotient space $\left\|X_{\bullet}\right\|$.

The simplicial de Rham complex. The de Rham complex $\left(\mathcal{A}_{d R}^{*}\left(X_{\bullet}\right), \delta\right)$ of $X_{\bullet}$ is given as the total complex of a double complex $\left(\mathcal{A}_{d R}^{*, *}\left(X_{\bullet}\right), \delta^{\prime}, \delta^{\prime \prime}\right)$ with

$$
\mathcal{A}_{d R}^{k}\left(X_{\bullet}\right)=\bigoplus_{r+s=k} \mathcal{A}_{d R}^{r, s}\left(X_{\bullet}\right)
$$

and $\delta=\delta^{\prime}+\delta^{\prime \prime}$, where $\mathcal{A}_{d R}^{r, s}\left(X_{\bullet}\right)=\Omega_{d R}^{s}\left(X_{r}\right)$ is the set of smooth complex $s$-forms on the smooth manifold $X_{r}$. Furthermore the differential

$$
\delta^{\prime \prime}: \mathcal{A}_{d R}^{r, s}\left(X_{\bullet}\right) \rightarrow \mathcal{A}_{d R}^{r, s+1}\left(X_{\bullet}\right)
$$

is the exterior differential on $\Omega_{d R}^{*}\left(X_{r}\right)$ and the differential

$$
\delta^{\prime}: \mathcal{A}_{d R}^{r, s}\left(X_{\bullet}\right) \rightarrow \mathcal{A}_{d R}^{r+1, s}\left(X_{\bullet}\right)
$$

is defined as the alternating sum

$$
\delta^{\prime}=\sum_{i=0}^{r+1}(-1)^{i} \varepsilon_{i}^{*} .
$$

The singular cochain complex. Given a commutative ring $R$ and a simplicial smooth manifold $X$ • we can also associate a singular cochain complex $\left(S^{*}\left(X_{\bullet} ; R\right), \partial\right)$. It is defined as a double complex $\left(S^{*, *}\left(X_{\bullet} ; R\right), \partial^{\prime}, \partial^{\prime \prime}\right)$ with

$$
S^{k}\left(X_{\bullet} ; R\right)=\bigoplus_{r+s=k} S^{r, s}\left(X_{\bullet} ; R\right)
$$

and $\partial=\partial^{\prime}+\partial^{\prime \prime}$, where

$$
S^{r, s}\left(X_{\bullet} ; R\right)=S^{s}\left(X_{r} ; R\right)
$$

is the set of singular cochains of degree $s$ on the smooth manifold $X_{r}$.

There is an integration map

$$
\mathcal{I}: \mathcal{A}_{d R}^{r, s}\left(X_{\bullet}\right) \rightarrow S^{r, s}\left(X_{\bullet} ; \mathbb{C}\right)
$$


which gives a morphism of double complexes and Dupont's general version of the de Rham theorem (see [D2], Proposition 6.1 for details) shows that this integration map induces natural isomorphisms

$$
H\left(\mathcal{A}_{d R}^{*}\left(X_{\bullet}, \delta\right)\right) \cong H\left(S^{*}\left(X_{\bullet}, \mathbb{C}\right), \partial\right) \cong H^{*}\left(\left\|X_{\bullet}\right\| ; \mathbb{C}\right) .
$$

Stoke's theorem gives that there is also a morphism of complexes

$$
\left.\mathcal{J}: \Omega_{d R}^{*}\left(X_{\bullet}\right), \mathrm{d}\right) \rightarrow\left(\mathcal{A}_{d R}^{*}\left(X_{\bullet}\right), \delta\right)
$$

defined on $\Omega_{d R}^{*}\left(\Delta^{n} \times X_{n}\right)$ by integration over the simplex $\Delta^{n}$

$$
\omega^{(n)} \in \Omega_{d R}^{*}\left(\Delta^{n} \times X_{n}\right) \mapsto \int_{\Delta^{n}} \omega^{(n)} .
$$

Theorem 2.3 with Corollary 2.8 of [D1] gives that this morphism is in fact a quasi-isomorphism, i.e.

$$
H\left(\Omega_{d R}^{*}\left(X_{\bullet}\right), \mathrm{d}\right) \cong H^{*}\left(\mathcal{A}_{d R}^{*}\left(X_{\bullet}\right), \delta\right) \cong H^{*}\left(\left\|X_{\bullet}\right\|, \mathbb{C}\right) .
$$

The singular cochain complex of compatible cochains. Let $R$ be a commutative ring. A compatible singular cochain $c$ on $X_{\bullet}$ is a sequence $\left\{c^{(n)}\right\}$ of cochains $c^{(n)} \in S^{k}\left(\Delta^{n} \times X_{n} ; R\right)$ satisfying the compatibility condition

$$
\left(\varepsilon^{i} \times i d\right)^{*} c^{(n)}=\left(i d \times \varepsilon_{i}\right)^{*} c^{(n-1)}
$$

in $S^{k}\left(\Delta^{n-1} \times X_{n}\right)$ for all $0 \leq i \leq n$ and all $n \geq 1$. Let $C^{k}\left(X_{\bullet} ; R\right)$ be the set of all compatible singular cochains on $X_{\bullet}$ and $\left(C^{*}\left(X_{\bullet} ; R\right), \mathrm{d}\right)$ be the singular cochain complex of compatible cochains.

It follows that the natural inclusion of cochain complexes

$$
\left(C^{*}\left(X_{\bullet} ; R\right) \mathrm{d}\right) \rightarrow\left(S^{*}\left(X_{\bullet} ; R\right), \partial\right)
$$

is a quasi-isomorphism [DHZ].

Integrating forms preserves the compatibility conditions and we therefore get an induced map of complexes [DHZ]

$$
\mathcal{I}^{\prime}: \Omega_{d R}^{*}\left(X_{\bullet}\right) \rightarrow C^{*}\left(X_{\bullet} ; \mathbb{C}\right)
$$

fitting into a commutative diagram

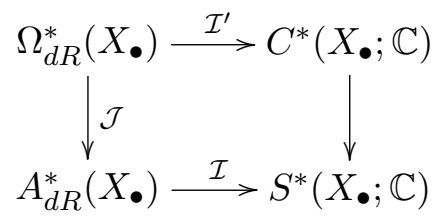

and which is again a quasi-isomorphism, i.e. we have

$$
H^{*}\left(\Omega_{d R}^{*}\left(X_{\bullet}\right), \mathrm{d}\right) \cong H^{*}\left(C^{*}\left(X_{\bullet} ; \mathbb{C}\right), \partial\right) .
$$

We will use these compatible de Rham and cochain complexes for the definition of multiplicative cohomology and differential characters of $X_{\bullet}$ in section 2 .

We recall finally the basic aspects of Chern-Weil theory in the simplicial context as developed in [D2] or [DHZ]. 
Principal bundles. Let $G$ be a Lie group. A principal $G$-bundle over a simplicial smooth manifold $X_{\bullet}$ is given by a simplicial smooth manifold $P_{\bullet}$ and a morphism $\pi_{\bullet}: P_{\bullet} \rightarrow X_{\bullet}$ of simplicial smooth manifolds, such that

(i) for each $n$ the map $\pi_{p}: P_{n} \rightarrow X_{n}$ is a principal $G$-bundle over $X_{n}$

(ii) for each morphism $f:[m] \rightarrow[n]$ of the simplex category $\Delta$ the induced map $f^{*}: P_{n} \rightarrow P_{m}$ is a morphism of $G$-bundles, i.e. we have a commutative diagram

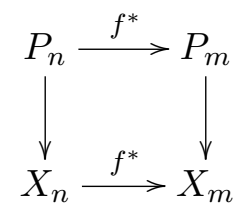

It follows, that if $\pi_{\bullet}: P_{\bullet} \rightarrow X_{\bullet}$ is a principal $G$-bundle over $X_{\bullet}$, then $\left|\pi_{\bullet}\right|:\left|P_{\bullet}\right| \rightarrow\left|X_{\bullet}\right|$ is a principal $G$-bundle with $G$-action induced by

$$
\Delta^{n} \times P_{n} \times G \rightarrow \Delta^{n} \times P_{n},(t, x, g) \mapsto(t, x g) .
$$

Connections and curvature on principal bundles. A connection $\theta$ on a principal $G$-bundle $\pi_{\bullet}: P_{\bullet} \rightarrow X$ over a simplicial manifold $X_{\bullet}$ is a $G$-invariant 1-form (in the de Rham complex of compatible forms)

$$
\theta \in \Omega_{d R}^{1}\left(P_{\bullet} ; \mathfrak{g}\right)
$$

taking values in the Lie algebra $\mathfrak{g}$ of $G$, on which $G$ acts via the adjoint representation, such that for each $n$ the restriction

$$
\theta^{(n)}=\left.\theta\right|_{\Delta^{n} \times P_{n}},
$$

is a connection on the bundle $\pi_{n}: \Delta^{n} \times P_{n} \rightarrow \Delta^{n} \times X_{n}$. So $\theta=\left\{\theta^{(n)}\right\}$ can as well be interpreted as a sequence of $\mathfrak{g}$-valued compatible 1 -forms.

The curvature $\Omega$ of the connection form $\theta$ is the differential form

$$
\Omega=d \theta+\frac{1}{2}[\theta, \theta] \in \Omega_{d R}^{2}\left(X_{\bullet} ; \mathfrak{g}\right) .
$$

We have the following general theorem concerning the Chern-Weil map of a simplicial smooth manifold ([D1], Proposition 3.7)

Theorem 1.1 (Dupont). Let $\Phi$ be an invariant polynomial. The differential form $\Phi(\theta) \in \Omega_{d R}^{*}\left(P_{\bullet}\right)$ is a closed form and descends to a closed form in $\Omega_{d R}^{*}\left(X_{\bullet}\right)$ and its cohomology class represents the image of the class $\Phi \in$ $H^{*}(B G ; \mathbb{C})$ under the Chern-Weil map

$$
H^{*}(B G ; \mathbb{C}) \rightarrow H^{*}(\|X \bullet\| ; \mathbb{C})
$$

associated to the principal bundle $\pi_{\bullet}: P_{\bullet} \rightarrow X_{\bullet}$.

With an abuse of notation, in the sequel we will denote also by $\Phi(\theta)$ the form in $\Omega_{d R}^{*}\left(X_{\bullet}\right)$.

In order to classify differential geometric invariants on simplicial smooth manifolds it is useful to extend the constructions outlined above to the 
category of bisimplicial smooth manifolds. This is straightforward and we will only briefly describe the constructions (compare also [DHZ] and [DJ]).

A bisimplicial smooth manifold $X_{\bullet \bullet}$ is a simplicial object in the category of simplicial smooth manifolds. In other words a bisimplicial smooth manifold is a functor

$$
X_{. \bullet}: \Delta^{o p} \times \Delta^{o p} \rightarrow\left(\mathcal{C}^{\infty}-\text { manifolds }\right) .
$$

We can think of $X_{\bullet \bullet}$ as a collection $X_{\bullet \bullet}=\left\{X_{m, n}\right\}$ of smooth manifolds $X_{m, n}$ for $m, n \geq 0$ together with smooth horizontal and vertical face and degeneracy maps

$$
\begin{aligned}
& \varepsilon_{i}^{\prime}: X_{m, n} \rightarrow X_{m-1, n}, \quad \varepsilon_{j}^{\prime \prime}: X_{m, n} \rightarrow X_{m, n-1} \\
& \eta_{i}^{\prime}: X_{m, n} \rightarrow X_{m+1, n}, \quad \eta_{j}^{\prime \prime}: X_{m, n} \rightarrow X_{m, n+1}
\end{aligned}
$$

for $0 \leq i \leq m$ and $0 \leq j \leq n$, where the horizontal and vertical maps commute and the usual simplicial identities hold horizontally and vertically.

The fat realization of a bisimplicial space $X_{\bullet \bullet}$ is the quotient space

$$
\| X \bullet \bullet=\coprod_{m, n \geq 0}\left(\Delta^{m} \times \Delta^{n} \times X_{m, n}\right) / \sim
$$

where the equivalence relation is generated by

$$
\left(\varepsilon^{i} \times i d \times i d\right)(t, s, x) \sim\left(i d \times i d \times \varepsilon_{i}^{\prime}\right)(t, s, x)
$$

for any $(t, s, x) \in \Delta^{m-1} \times \Delta^{n} \times X_{m, n}$ and

$$
\left(i d \times \varepsilon^{j} \times i d\right)(t, s, x) \sim\left(i d \times i d \times \varepsilon_{j}^{\prime \prime}\right)(t, s, x)
$$

for any $(t, s, x) \in \Delta^{m} \times \Delta^{n-1} \times X_{m, n}$.

In a similar manner as for simplicial smooth manifolds, we can associate two de Rham complexes and a singular cochain complex for bisimplicial smooth manifolds.

The de Rham complex of compatible forms. A bisimplicial smooth $k$-form $\omega$ on $X_{\bullet \bullet}$ is a sequence $\left\{\omega^{(m, n)}\right\}$ of smooth complex $k$-forms

$$
\omega^{(m, n)} \in \Omega_{d R}^{k}\left(\Delta^{m} \times \Delta^{n} \times X_{m, n}\right)
$$

satisfying the compatibility conditions

$$
\left(\varepsilon^{i} \times i d \times i d\right)^{*} \omega^{(m, n)}=\left(i d \times \mathrm{id} \times \varepsilon_{\mathrm{i}}^{\prime}\right)^{*} \omega^{(\mathrm{m}-1, \mathrm{n})}
$$

in $\Omega_{d R}^{k}\left(\Delta^{m-1} \times \Delta^{n} \times X_{m, n}\right)$ for all $0 \leq i \leq m, m \geq 1$ and $n \geq 0$ as well as the compatibility conditions

$$
\left(i d \times \varepsilon^{j} \times i d\right)^{*} \omega^{(m, n)}=\left(i d \times i d \times \varepsilon_{j}^{\prime \prime}\right)^{*} \omega^{(m-1, n)}
$$

in $\Omega_{d R}^{k}\left(\Delta^{m} \times \Delta^{n-1} \times X_{m, n}\right)$ for all $0 \leq j \leq n, n \geq 1$ and $m \geq 0$.

We denote the set of bisimplicial smooth $k$-forms by $\Omega_{d R}^{k}\left(X_{\bullet \bullet}\right)$. The exterior differential on $\Omega_{d R}^{*}\left(\Delta^{m} \times \Delta^{n} \times X_{m, n}\right)$ induces an exterior differential $\mathrm{d}$ on $\Omega_{d R}^{*}\left(X_{\bullet \bullet}\right)$ and we get a complex $\left(\Omega_{d R}^{*}\left(X_{\bullet \bullet}\right), \mathrm{d}\right)$, the de Rham complex of compatible forms on $X$.. 
We note also that we can view the complex $\left(\Omega_{d R}^{*}\left(X_{\bullet \bullet}\right), \mathrm{d}\right)$ as a triple complex $\left(\Omega_{d R}^{*, *, *}\left(X_{\bullet \bullet}\right), \mathrm{d}_{\Delta}^{\prime}, \mathrm{d}_{\Delta}^{\prime \prime}, \mathrm{d}_{X}\right)$ with

$$
\Omega_{d R}^{k}\left(X_{\bullet \bullet}\right)=\bigoplus_{r+s+t} \Omega_{d R}^{r, s, t}\left(X_{\bullet \bullet}\right)
$$

and $\mathrm{d}=\mathrm{d}_{\Delta}^{\prime}+\mathrm{d}_{\Delta}^{\prime \prime}+\mathrm{d}_{X}$ where $\Omega_{d R}^{r, s, t}\left(X_{\bullet \bullet}\right)$ is the complex vector space of $(r+s+t)$-forms, which when restricted to $\Delta^{m} \times \Delta^{n} \times X_{m, n}$ are locally of the form

$$
\begin{gathered}
\left.a\right|_{\Delta^{m} \times \Delta^{n} \times X_{m, n}}=\sum a_{i_{1} \ldots i_{r} j_{1} \ldots j_{s} k_{1} \ldots k_{t}} \mathrm{~d} t_{i_{1}} \wedge \ldots \wedge \mathrm{d} t_{i_{r}} \wedge \mathrm{d} s_{j_{1}} \wedge \ldots \\
\ldots \wedge \mathrm{d} s_{j_{s}} \wedge \mathrm{d} x_{k_{1}} \wedge \ldots \wedge \mathrm{d} x_{k_{t}}
\end{gathered}
$$

with $\left(t_{0}, \ldots, t_{m}\right)$ and $\left(s_{0}, \ldots, s_{n}\right)$ the barycentric coordinates of $\Delta^{m}$ and $\Delta^{n}$ respectively and the $\left\{x_{k}\right\}$ are local coordinates of $X_{m, n}$.

The simplicial de Rham complex. Again we also have the simplicial de Rham complex $\left(\mathcal{A}^{*}\left(X_{\bullet \bullet}\right), \delta\right)$ of $X_{\bullet \bullet}$ given as the total complex of the triple complex $\left(\mathcal{A}_{d R}^{*, *, *}\left(X_{\bullet \bullet}\right), \delta^{\prime}, \delta^{\prime \prime}, \delta^{\prime \prime \prime}\right)$ with

$$
\mathcal{A}_{d R}^{k}\left(X_{\bullet \bullet}\right)=\bigoplus_{r+s+t=k} \mathcal{A}_{d R}^{r, s, t}\left(X_{\bullet \bullet}\right)
$$

with

and $\delta=\delta^{\prime}+\delta^{\prime \prime}+\delta^{\prime \prime \prime}$.

$$
\mathcal{A}_{d R}^{r, s, t}\left(X_{\bullet \bullet}\right)=\Omega_{d R}^{t}\left(X_{r, s}\right)
$$

The singular cochain complex. For a commutative ring $R$, we similarly define the singular cochain complex $\left(S^{*}\left(X_{\bullet \bullet} ; R\right), \partial\right)$ of $X_{\bullet \bullet}$ given as the total complex of the triple complex $\left(S^{*, * *}\left(X_{\bullet \bullet} ; R\right), \partial^{\prime}, \partial^{\prime \prime}, \partial^{\prime \prime \prime}\right)$ with

$$
S^{k}\left(X_{\bullet \bullet} ; R\right)=\bigoplus_{r+s+t=k} S^{r, s, t}\left(X_{\bullet \bullet} ; R\right)
$$

with

$$
S^{r, s, t}\left(X_{\bullet \bullet} ; R\right)=S^{t}\left(X_{r, s} ; R\right)
$$

and $\partial=\partial^{\prime}+\partial^{\prime \prime}+\partial^{\prime \prime \prime}$.

Using iteratively the arguments as in the case for simplicial smooth manifolds, we can finally also derive a de Rham theorem relating the cohomology of all the complexes defined with the cohomology of the realization of $X_{\bullet \bullet}$, i.e. we have natural isomorphisms

$$
H\left(\Omega_{d R}^{*}\left(X_{\bullet \bullet}\right), \mathrm{d}\right) \cong H^{*}\left(\mathcal{A}_{d R}^{*}\left(X_{\bullet \bullet}\right), \delta\right) \cong H^{*}\left(\left\|X_{\bullet \bullet}\right\|, \mathbb{C}\right) .
$$

We remark that we can also define again the singular cochain complex of compatible forms $C^{*}\left(X_{\bullet \bullet} ; R\right)$ in a same way as for $X_{\bullet}$ using two compatibility conditions instead. Again we have quasi-isomorphisms as in the simplicial case between the various complexes.

Finally we can extend the elements of simplicial Chern-Weil theory to bisimplicial smooth manifolds, especially we remark that we can define principal $G$-bundles $\pi_{\bullet \bullet}: P_{\bullet \bullet} \rightarrow X_{\bullet \bullet}$ for the action of a Lie group $G$ and a 
connection $\theta$ on $\pi_{\bullet \bullet}$ which is again a 1 -form

$$
\theta \in \Omega_{d R}^{1}\left(P_{\bullet \bullet} ; \mathfrak{g}\right) \text {. }
$$

The curvature $\Omega$ of the connection form $\nabla$ is again the differential form

$$
\Omega=d \theta+\frac{1}{2}[\theta, \theta] \in \Omega_{d R}^{2}\left(X_{\bullet \bullet} ; \mathfrak{g}\right) .
$$

Again a version of Dupont's theorem (Theorem 1.1) holds in the context of bisimplicial manifolds. When defining characteristic classes we will need that given any connection on a principal bundle, we can construct a connection on (a model of) the universal bundle that pulls back to the given one. For the convenience of the reader, we recall the theorem stating this fact and outline its proof, which for $G l_{n}(\mathbb{C})$-principal bundles is Proposition 6.15 of [DHZ].

Theorem 1.2. Let $G$ be a Lie group, $X \bullet$ a simplicial smooth manifold and $\pi_{\bullet}: P_{\bullet} \rightarrow X_{\bullet}$ a principal $G$-bundle with connection

$$
\theta \in \Omega_{d R}^{1}\left(P_{\bullet} ; \mathfrak{g}\right) .
$$

Then there exists a bisimplicial smooth manifold $B_{\text {.. }}$ of the homotopy type of the classifying space $B G$ and a $G$-principle bundle $U_{\bullet \bullet} \rightarrow B_{\bullet \bullet}$ with a connection $\theta_{U_{\bullet \bullet}} \in \Omega_{d R}^{1}\left(U_{\bullet \bullet} ; \mathfrak{g}\right)$ and a morphism $(\Psi, \psi)$ of $G$-bundles

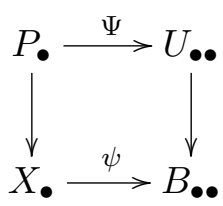

such that $\Psi^{*}\left(\theta_{U . \bullet}\right)=\theta$.

Proof. We define the bisimplicial manifold $U_{\bullet \bullet}$ as follows:

$$
U_{\bullet} m=\left(P_{\bullet}\right)^{m+1}
$$

with face maps

$$
\begin{gathered}
d_{i}: U_{\bullet m} \rightarrow U_{\bullet m-1} \\
\left(u_{0}, \ldots u_{m}\right) \mapsto\left(u_{0}, \ldots, u_{i-1}, u_{i+1}, \ldots u_{m}\right) \text { for } 0 \leq \mathrm{i} \leq \mathrm{m}
\end{gathered}
$$

and degeneracy maps

$$
\begin{gathered}
s_{i}: U_{\bullet m} \rightarrow U_{\bullet m+1} \\
\left(u_{0}, \ldots u_{m}\right) \mapsto\left(u_{0}, \ldots, u_{i-1}, u_{i}, u_{i}, u_{i+1} \ldots u_{m}\right) \text { for } 0 \leq \mathrm{i} \leq \mathrm{m} .
\end{gathered}
$$

The fat realization $\left\|U_{\bullet \bullet}\right\|$ of this simplicial manifold is contractible, i.e. homotopy equivalent to a point $[\mathrm{S}]$. Now the free $G$-action on $P_{\bullet}$ induces a free $G$-action on $U_{\bullet . .}$. We define the classifying bisimplicial smooth manifold as the quotient

$$
B_{\bullet \bullet}=U_{\bullet \bullet} / G
$$

We get a principal $G$-bundle $U_{\bullet \bullet} \rightarrow B \bullet \bullet$, the universal principle $G$-bundle and $\|B . \bullet\|$ is homotopy eqivalent to the classifying space $B G$ of $G$. 
We define now the connection $\theta_{U_{\bullet \bullet}} \in \Omega_{d R}^{1}\left(U_{\bullet \bullet} ; \mathfrak{g}\right)$ on the universal principal $G$-bundle by the compatible sequence $\left\{\theta_{U_{\bullet \bullet}}^{(p)}\right\}$ defined as

$$
\theta_{U_{\bullet \bullet}}^{(p)}=\sum_{j=0}^{p} t_{j} p r_{j}^{*}(\theta) \in \Omega_{d R}^{1}\left(\Delta^{p} \times U_{\bullet p} ; \mathfrak{g}\right)
$$

where $\left(t_{0}, \ldots, t_{p}\right)$ are the barycentric coordinates of $\Delta^{p}$ and $p r_{j}: U_{\bullet p} \rightarrow U_{\bullet 0}$ the canonical projections.

The canonical isomorphism of simplicial manifolds $P_{\bullet} \rightarrow U_{\bullet} 0$ gives a $G$ equivariant map

$$
\Psi: P_{\bullet} \rightarrow U_{\bullet \bullet}
$$

and induces a map

$$
\psi: X_{\bullet}=P_{\bullet} / G \rightarrow B_{\bullet \bullet}=U_{\bullet \bullet} / G
$$

such that $(\Psi, \psi)$ pulls back the principal $G$-bundle $P_{\bullet}$ over $X_{\bullet}$ and the connection $\theta$ as stated in the theorem.

\section{Multiplicative Cohomology and Differential Characters}

We will now define general versions of Karoubi's multiplicative cohomology and Cheeger-Simons differential characters for smooth simplicial manifolds with respect to any given filtration of the simplicial de Rham complex. As a special case with respect to the 'filtration bête' we will recover the group of Cheeger-Simons differential characters for smooth simplicial manifolds as introduced in [DHZ].

In general, for a given complex $C^{*}$ let $\sigma_{\geq p} C^{*}$ denote the filtration via truncation in degrees below $p$ and similarly $\sigma_{<p} C^{*}$ denotes truncation of $C^{*}$ in degrees greater or equal $p$. Let us first consider the special case of Deligne's 'filtration bête' [De] for the simplicial de Rham complex $\Omega_{d R}^{*}\left(X_{\bullet}\right)$ of a simplicial manifold $X_{\bullet}$. The 'filtration bête' $\sigma=\left\{\sigma_{\geq p} \Omega_{d R}^{*}\left(X_{\bullet}\right)\right\}$ is given as truncation in degrees below $p$

$$
\sigma_{\geq p} \Omega_{d R}^{j}\left(X_{\bullet}\right)= \begin{cases}0 & j<p, \\ \Omega_{d R}^{j}\left(X_{\bullet}\right) & j \geq p\end{cases}
$$

We define the group of Cheeger-Simons differential characters as follows:

Definition 2.1. (See [DHZ]). Let $X$ • be a simplicial smooth manifold and $\Lambda$ be a subgroup of $\mathbb{C}$. The group of $(\bmod \Lambda)$ differential characters of degree $k$ of $X_{\bullet}$ is given by

$$
\hat{H}^{k-1}\left(X_{\bullet} ; \mathbb{C} / \Lambda\right)=H^{k}\left(\operatorname{cone}\left(\sigma_{\geq k} \Omega_{d R}^{*}\left(X_{\bullet}\right) \rightarrow C^{*}\left(X_{\bullet} ; \mathbb{C} / \Lambda\right)\right)\right) .
$$

Now let $\mathcal{F}=\left\{F^{r} \Omega_{d R}^{*}\left(X_{\bullet}\right)\right\}$ be any given filtration of the simplicial de Rham complex. We define the multiplicative cohomology groups of $X \bullet$ with respect to $\mathcal{F}$ as follows: 
Definition 2.2. Let $X_{\bullet}$ be a simplicial smooth manifold, $\Lambda$ be a subgroup of $\mathbb{C}$ and $\mathcal{F}=\left\{F^{r} \Omega_{d R}^{*}\left(X_{\bullet}\right)\right\}$ be a filtration of $\Omega_{d R}^{*}\left(X_{\bullet}\right)$. The multiplicative cohomology groups of $X$ • associated to the filtration $\mathcal{F}$ are given by

$$
M H_{n}^{2 r}\left(X_{\bullet} ; \Lambda ; \mathcal{F}\right)=H^{2 r-n}\left(\operatorname{cone}\left(C^{*}\left(X_{\bullet} ; \Lambda\right) \oplus F^{r} \Omega_{d R}^{*}\left(X_{\bullet}\right) \rightarrow C^{*}\left(X_{\bullet} ; \mathbb{C}\right)\right)\right) .
$$

In order to be be able to introduce secondary charactersitic classes for connections whose curvature and characteristic forms lie in a filtration of the simplicial de Rham complex we introduce a more general version of differential characters associated to any given filtration. For smooth manifolds these invariants were studied systematically by the first author in $[\mathrm{F}]$.

Definition 2.3. Let $X_{\bullet}$ be a simplicial smooth manifold, $\Lambda$ be a subgroup of $\mathbb{C}$ and $\mathcal{F}=\left\{F^{r} \Omega_{d R}^{*}\left(X_{\bullet}\right)\right\}$ be a filtration of $\Omega_{d R}^{*}\left(X_{\bullet}\right)$. The groups of differential characters $(\bmod \Lambda)$ of degree $k$ of $X$ • associated to the filtration $\mathcal{F}$ are given by

$$
\hat{H}_{r}^{k-1}\left(X_{\bullet} ; \mathbb{C} / \Lambda ; \mathcal{F}\right)=H^{k}\left(\operatorname{cone}\left(\sigma_{\geq k} F^{r} \Omega_{d R}^{*}\left(X_{\bullet}\right) \rightarrow C^{*}\left(X_{\bullet} ; \mathbb{C} / \Lambda\right)\right)\right) .
$$

The truncation in degrees below $k$ of a complex which is already truncated in degrees below $k$ leaves it unchanged, hence if $\mathcal{F}$ is Deligne's 'filtration bête' of $\Omega^{*}\left(X_{\bullet}\right)$, we recover the ordinary groups of differential characters of $X_{\bullet}$ as in Definition 2.1.

We have the following main theorem generalizing Theorem 2.3 of $[\mathrm{F}]$.

Theorem 2.4. Let $X_{\bullet}$ be a simplicial smooth manifold, $\Lambda$ be a subgroup of $\mathbb{C}$ and $\mathcal{F}=\left\{F^{r} \Omega_{d R}^{*}\left(X_{\bullet}\right)\right\}$ be a filtration of $\Omega_{d R}^{*}\left(X_{\bullet}\right)$. There exists a surjective map

$$
\Xi: \hat{H}_{r}^{2 r-n-1}\left(X_{\bullet} ; \mathbb{C} / \Lambda ; \mathcal{F}\right) \rightarrow M H_{n}^{2 r}\left(X_{\bullet} ; \Lambda ; \mathcal{F}\right)
$$

whose kernel is the group of forms in $F^{r} \Omega_{d R}^{2 r-n-1}\left(X_{\bullet}\right)$ modulo those forms that are closed and whose complex cohomology class is the image of a class in $H^{*}\left(X_{\bullet} ; \Lambda\right)$.

Proof: Let $\mathcal{A}\left(F^{r}\right)$ and $\mathcal{B}\left(F^{r}\right)$ denote the cone complexes used in the definition of the groups of differential characters and multiplicative cohomology associated to the filtration $\mathcal{F}$, i.e.

$$
\begin{gathered}
\mathcal{A}\left(F^{r}\right)=\operatorname{cone}\left(\sigma_{\geq k} F^{r} \Omega_{d R}^{*}\left(X_{\bullet}\right) \rightarrow C^{*}\left(X_{\bullet} ; \mathbb{C} / \Lambda\right)\right) \\
\mathcal{B}\left(F^{r}\right)=\operatorname{cone}\left(C^{*}\left(X_{\bullet} ; \Lambda\right) \oplus F^{r} \Omega_{d R}^{*}\left(X_{\bullet}\right) \rightarrow C^{*}\left(X_{\bullet} ; \mathbb{C}\right)\right)
\end{gathered}
$$

There is a quasi-isomorphism between the cone complexes

$$
\begin{gathered}
\operatorname{cone}\left(\sigma_{\geq k} F^{r} \Omega_{d R}^{*}\left(X_{\bullet}\right) \rightarrow C^{*}\left(X_{\bullet} ; \mathbb{C} / \Lambda\right)\right) \\
\operatorname{cone}\left(C^{*}\left(X_{\bullet} ; \Lambda\right) \oplus \sigma_{\geq k} F^{r} \Omega_{d R}^{*}\left(X_{\bullet}\right) \rightarrow C^{*}\left(X_{\bullet} ; \mathbb{C}\right)\right)
\end{gathered}
$$

and we get a short exact sequence of complexes

$$
0 \rightarrow \mathcal{A}\left(F^{r}\right) \rightarrow \mathcal{B}\left(F^{r}\right) \rightarrow \sigma_{<k} F^{r} \Omega_{d R}^{*}\left(X_{\bullet}\right) \rightarrow 0
$$

where $\sigma_{<k}$ denotes truncation in degrees greater or equal to $k$. The statement follws now from the long exact sequence in cohomology associated to 
this short exact sequences of complexes, because for $k=2 r-n$ the cohomology group $H^{2 r-n}\left(\sigma_{<2 r-n} F^{r} \Omega_{d R}^{*}\left(X_{\bullet}\right)\right)$ is trivial.

We can identify the classical Cheeger-Simons differential characters with multiplicative cohomology groups as follows

Corollary 2.5. Let $X_{\bullet}$ be a simplicial smooth manifold and $\Lambda$ be a subgroup of $\mathbb{C}$. There is an isomorphism

$$
\hat{H}^{r-1}\left(X_{\bullet} ; \mathbb{C} / \Lambda\right) \cong M H_{r}^{2 r}\left(X_{\bullet} ; \Lambda ; \sigma\right)
$$

Proof. This is a direct consequence of Theorem 2.4 in the case when $n=r$ and the filtration $\mathcal{F}$ is Deligne's 'filtration bête' using the definitions and the quasi-isomorphism of complexes mentioned in the proof of Theorem 2.4. above

In Karoubi's original approach [K2], [K3] towards multiplicative cohomology and differential characters for a smooth manifold $M$ the complex of modified singular cochains

$$
\tilde{C}^{*}(M ; \mathbb{Z})=\operatorname{cone}\left(\Omega_{d R}^{*}(M) \times S^{*}(M ; \mathbb{Z}) \rightarrow S^{*}(M ; \mathbb{C})\right)
$$

is used instead. However this complex is chain homotopy equivalent to the usual complex of (smooth) singular cochains $S^{*}(M ; \mathbb{Z})$ of $M$. Again, also in the more general case of a simplicial smooth manifold $X \bullet$ we can define the complex of modified compatible cochains $\tilde{C}^{*}\left(X_{\bullet} ; \mathbb{Z}\right)$ as

$$
\tilde{C}^{*}\left(X_{\bullet} ; \mathbb{Z}\right)=\operatorname{cone}\left(\Omega_{d R}^{*}\left(X_{\bullet}\right) \times C^{*}\left(X_{\bullet} ; \mathbb{Z}\right) \rightarrow C^{*}\left(X_{\bullet} ; \mathbb{C}\right)\right)
$$

and proceed as in $[\mathrm{K} 3]$ or $[\mathrm{F}]$ for the definition of multiplicative cohomology. But we can then show that the resulting complex using modified cochains is quasi-isomorphic to the compatible cochain complex $C^{*}\left(X_{\bullet} ; \mathbb{Z}\right)$ used here and the resulting cohomology groups are isomorphic to the ones defined above.

As in the manifold case, it can be shown that the multiplicative cohomology groups fit in the following long exact sequence (compare [K3])

$$
\begin{array}{r}
\ldots \rightarrow H^{2 r-n-1}\left(\left\|X_{\bullet}\right\| ; \Lambda\right) \rightarrow H^{2 r-n-1}\left(\Omega_{d R}^{*}\left(X_{\bullet} / F^{r} \Omega_{d R}^{*}\left(X_{\bullet}\right)\right)\right) \rightarrow \\
\rightarrow M H_{n}^{2 r}\left(X_{\bullet} ; \Lambda ; \mathcal{F}\right) \rightarrow \ldots
\end{array}
$$

The groups of differential characters fit also in short exact sequences analogous to the ones in $[\mathrm{CS}]$, which are again a special case of the one above.

Remark 2.6. There are several equivalent conventions for the cone of a map of complexes $f^{*}: A^{*} \rightarrow B^{*}$. Throughout this paper we will use the following: cone $\left(f^{*}\right)^{n}=A^{n} \oplus B^{n-1}$ with differential given by $\mathrm{d}(a, b)=$ $\left(\mathrm{d}_{A} a,(-1)^{n+1} f^{n}(a)+\mathrm{d}_{B} b\right)$, where $a \in A^{n}, b \in B^{n-1}$ and $\mathrm{d}_{A}, \mathrm{~d}_{B}$ are the differentials in the complexes $A^{*}, B^{*}$ respectively.

We will discuss briefly some applications to specific examples of simplicial smooth manifolds. In order to deal with them in a unified way, we briefly recall the notion of a nerve for a topological category $[\mathrm{S}],[\mathrm{D} 1],[\mathrm{D} 2]$. 
Let $\mathcal{C}$ be a topological category, i. e. a small category such that the set of objects $O b(\mathcal{C})$ and the set of morphisms $\operatorname{Mor}(\mathcal{C})$ are both topological spaces such that

(i) the source and target maps

$$
s, t: \operatorname{Mor}(\mathcal{C}) \rightarrow O b(\mathcal{C})
$$

are continuous maps.

(ii) composition of arrows is continuous, i. e. if

$$
\operatorname{Mor}(\mathcal{C})^{\circ} \subseteq \operatorname{Mor}(\mathcal{C}) \times \operatorname{Mor}(\mathcal{C})
$$

is the set of pairs $\left(f, f^{\prime}\right)$ with $s(f)=t\left(f^{\prime}\right)$, then the composition map $\operatorname{Mor}(\mathcal{C})^{\circ} \rightarrow \operatorname{Mor}(\mathcal{C})$ is a continuous map.

Associated to a topological category is a simplicial space $\mathcal{N}(\mathcal{C}) \bullet=\left\{\mathcal{N}(\mathcal{C})_{n}\right\}$, the nerve of the category $\mathcal{C}$. We have

$$
\mathcal{N}(\mathcal{C})_{0}=\operatorname{Ob}(\mathcal{C}), \mathcal{N}(\mathcal{C})_{1}=\operatorname{Mor}(\mathcal{C}), \mathcal{N}(\mathcal{C})_{2}=\operatorname{Mor}(\mathcal{C})^{\circ}
$$

and in general

$$
\mathcal{N}(\mathcal{C})_{n} \subseteq \operatorname{Mor}(\mathcal{C}) \times \ldots \operatorname{Mor}(\mathcal{C})(\text { ntimes })
$$

is the subset of composable strings of morphisms

$$
\bullet \stackrel{f_{1}}{\leftarrow} \bullet \stackrel{f_{2}}{\leftarrow} \bullet \leftarrow \bullet \cdots \bullet \stackrel{f_{n}}{\leftarrow} \bullet,
$$

i. e. an $n$-tupel $\left(f_{1}, f_{2}, \ldots, f_{n}\right) \in \mathcal{N}(\mathcal{C})_{n}$ if and only if $s\left(f_{i}\right)=t\left(f_{i+1}\right)$ for all $1 \leq i \leq n-1$.

The face maps $\varepsilon_{i}: \mathcal{N}(\mathcal{C})_{n} \rightarrow \mathcal{N}(\mathcal{C})_{n-1}$ are given as

$$
\varepsilon_{i}\left(f_{1}, f_{2}, \ldots, f_{n}\right)= \begin{cases}\left(f_{2}, \ldots, f_{n}\right) & i=0, \\ \left(f_{1}, \ldots, f_{i} \circ f_{i+1}, \ldots, f_{n}\right) & 0<i<n, \\ \left(f_{1}, \ldots, f_{n-1}\right) & i=n .\end{cases}
$$

The degeneracy maps $\eta_{i}: \mathcal{N}(\mathcal{C})_{n} \rightarrow \mathcal{N}(\mathcal{C})_{n-1}$ are given as

$$
\eta_{i}\left(f_{1}, \ldots, f_{n}\right)=\left(f_{1}, \ldots, f_{i-1}, i d, f_{i}, \ldots, f_{n}\right), 0 \leq i \leq n .
$$

The nerve $\mathcal{N}$ is a functor from the category of topological categories and continuous functors to the category of simplicial spaces.

Classifying spaces of Lie groups. Let $G$ be a Lie group viewed as a topological category with one object, i.e.

$$
\operatorname{Ob}(G)=*, \operatorname{Mor}(G)=G .
$$

Furthermore let $\bar{G}$ be the topological category defined as

$$
\operatorname{Ob}(\bar{G})=G, \operatorname{Mor}(\bar{G})=G \times G .
$$

There is an obvious functor

$$
\gamma: \bar{G} \rightarrow G, \gamma\left(g_{0}, g_{1}\right)=g_{0} g_{1}^{-1}
$$

inducing a map

$$
\gamma: \mathcal{N}(\bar{G}) \bullet \mathcal{N}(G) \bullet, \gamma\left(g_{0}, \ldots g_{n}\right)=\left(g_{0} g_{1}^{-1}, \ldots, g_{n-1} g_{n}^{-1}\right)
$$


between simplicial smooth manifolds and applying the fat realization functor gives the universal principal $G$-bundle

$$
\gamma_{G}: E G \rightarrow B G .
$$

Using the simplicial smooth manifold $\mathcal{N}(G)$ • we can now define

Definition 2.7. Let $G$ be a Lie group, $\Lambda$ a subgroup of $\mathbb{C}$ and let $\mathcal{F}=$ $\left\{F^{r} \Omega_{d R}^{*}\left(\mathcal{N}(G)_{\bullet}\right\}\right.$ be a filtration of $\Omega_{d R}^{*}\left(\mathcal{N}(G)_{\bullet}\right)$. The multiplicative cohomology groups of $B G$ associated to the filtration $\mathcal{F}$ are defined as

$$
M H_{n}^{2 r}(B G, \Lambda, \mathcal{F})=M H_{n}^{2 r}(\mathcal{N}(G) \bullet, \Lambda, \mathcal{F})
$$

and the group of differential characters as

$$
\hat{H}_{r}^{k-1}(B G, \mathbb{C} / \Lambda, \mathcal{F})=\hat{H}_{r}^{k-1}(\mathcal{N}(G) \bullet, \mathbb{C} / \Lambda, \mathcal{F}) .
$$

As in the general case we get the identification from Corollary 2.5 in the case of the 'filtration bête' $\sigma$

$$
\hat{H}^{r-1}(B G, \mathbb{C} / \Lambda) \cong M H_{r}^{2 r}(B G, \Lambda, \sigma) .
$$

for the classical Cheeger-Simons differential characters. These invariants were studied in the case $G=G L_{n}(\mathbb{C})$ already by Dupont, Hain and Zucker [DHZ].

We can generalize this situation much further in the following way.

Classifying spaces of Lie groupoids. Let $\mathcal{G}: X_{1} \rightrightarrows X_{0}$ be a Lie groupoid, i.e. both the set of objects $X_{0}$ and the set of morphisms $X_{1}$ are $\mathcal{C}^{\infty}$-manifolds and all structure maps are smooth and the source and traget maps are both smooth submersions.

As in the example above we can apply the nerve functor to the category $\mathcal{G}$ and we get again a simplicial smooth manifold $X_{\bullet}=\mathcal{N}(\mathcal{G}) \bullet$ where

$$
X_{n}=X_{1} \times_{X_{0}} X_{1} \times \cdots \times_{X_{0}} X_{1} \text { (n factors). }
$$

Let $B \mathcal{G}$ be the classifying space of $\mathcal{G}$, i. e. the fat realization of the nerve $B \mathcal{G}=\left\|\mathcal{N}\left(\mathcal{G}_{\bullet}\right)\right\|$. We define

Definition 2.8. Let $\mathcal{G}: X_{1} \rightrightarrows X_{0}$ be a Lie groupoid, $\Lambda$ a subgroup of $\mathbb{C}$ and let $\mathcal{F}=\left\{F^{r} \Omega_{d R}^{*}\left(\mathcal{N}(\mathcal{G})_{\bullet}\right\}\right.$ be a filtration of $\Omega_{d R}^{*}\left(\mathcal{N}(\mathcal{G})_{\bullet}\right)$. The multiplicative cohomology groups of $B \mathcal{G}$ associated to the filtration $\mathcal{F}$ are defined as

$$
M H_{n}^{2 r}(B \mathcal{G}, \Lambda, \mathcal{F})=M H_{n}^{2 r}(\mathcal{N}(\mathcal{G}) \bullet, \Lambda, \mathcal{F})
$$

and the group of differential characters as

$$
\hat{H}_{r}^{k-1}(B \mathcal{G}, \mathbb{C} / \Lambda, \mathcal{F})=\hat{H}_{r}^{k-1}(\mathcal{N}(\mathcal{G}) ., \mathbb{C} / \Lambda, \mathcal{F}) .
$$

Actions of Lie groups on smooth manifolds. Let $X$ be a $\mathcal{C}^{\infty}$ manifold and $G$ a Lie group which acts smoothly from the left on $X$. We have a Lie groupoid

$$
\mathcal{G}: G \times X \rightrightarrows X
$$


with source map $s: G \times X \rightarrow X, s(g, x)=x$, target map $t: G \times X \rightarrow$ $X, t(g, x)=g x$ and composition map

$$
m:(G \times X) \times{ }_{X}(G \times X) \rightarrow G \times X,(g, h x)(h, x)=(g h, x) .
$$

This Lie groupoid was studied in detail by Getzler $[\mathrm{G}]$ in order to define an equivariant version of the classical Chern character. Applying the nerve functor again gives a simplicial manifold, the homotopy quotient, which allows us to define equivariant versions of the multiplicative cohomology invariants

Definition 2.9. Let $G$ be a Lie group, acting smoothly on a smooth manifold $X, \Lambda$ a subgroup of $\mathbb{C}$ and let $\mathcal{F}=\left\{F^{r} \Omega_{d R}^{*}(\mathcal{N}(G \times X \rightrightarrows X)\right.$.$\} be a$ filtration of $\Omega_{d R}^{*}(\mathcal{N}(G \times X \rightrightarrows X) \bullet)$. The equivariant multiplicative cohomology groups of $X$ associated to the filtration $\mathcal{F}$ are defined as

$$
M H_{G}^{2 r, n}(X, \Lambda, \mathcal{F})=M H_{n}^{2 r}(\mathcal{N}(G \times X \rightrightarrows X) ., \Lambda, \mathcal{F})
$$

and the group of equivariant differential characters as

$$
\hat{H}_{G}^{k-1, r}(X, \mathbb{C} / \Lambda, \mathcal{F})=\hat{H}_{r}^{k-1}(\mathcal{N}(G \times X \rightrightarrows X) ., \mathbb{C} / \Lambda, \mathcal{F}) .
$$

We will study secondary theories for classifying spaces of Lie groupoids and Lie groups in more detail in further papers in view of applications to foliations, differentiable orbifolds and differentiable stacks. Equivariant differential characters for orbifolds of type $[M / G]$ for a smooth manifold $M$ with smooth action of a Lie group $G$ with finite stabilizers were constructed and studied systematically by Lupercio and Uribe [LU]. Their approach follows closely the modified definition of Cheeger-Simons cohomology due to Hopkins and Singer [HS]. It would be interesting to study the relation of these invariants with the ones defined here, especially for different filtrations of the de Rham complex. Chern-Weil theory for principal $G$-bundles over a Lie groupoid was systematically developped by Laurent-Gengoux, Tu and $\mathrm{Xu}$ [LTX]. This framework can be applied to differentiable stacks using the general de Rham cohomology of differentiable stacks as developped by Behrend $[\mathrm{B}]$. The framework developped here allows the definition of multiplicative cohomology groups and groups of differential characters for arbitrary filtrations of the de Rham complex of a differentiable stack, which will be the topic of a sequel to this paper.

\section{Multiplicative Bundles And MUltiplicative K-Theory}

Let $G$ be a Lie group and $\theta_{0}, \ldots, \theta_{q}$ be connections on the principal $G$ bundle $\pi_{\bullet}: P_{\bullet} \rightarrow X_{\bullet}$, i.e.

$$
\theta_{j} \in \Omega_{d R}^{1}\left(P_{\bullet} ; \mathfrak{g}\right)
$$

such that for all $p$ and all $0 \leq j \leq q$

$$
\theta_{j}^{(p)} \in \Omega_{d R}^{1}\left(\Delta^{p} \times P_{p} ; \mathfrak{g}\right)
$$


i.e. the restrictions $\theta_{j}^{(p)}$ are connections on the bundle

$$
\Delta^{p} \times P_{p} \rightarrow \Delta^{p} \times X_{p} .
$$

Fix $q$ and let $\Delta^{q}$ be the standard simplex in $\mathbb{R}^{q+1}$ parametrized by coordinates $\left(s_{0}, \ldots, s_{q}\right)$.

Lemma 3.1. The form $\sum_{j=0}^{q} \theta_{j} s_{j}$ defines a (partial) connection on the pullback bundle $\pi^{*} P_{\bullet} \rightarrow X_{\bullet} \times \Delta^{q}$ where $\pi: X_{\bullet} \times \Delta^{q} \rightarrow X_{\bullet}$ is the projection.

Proof. For each $m$ the sum $\left(\sum_{j=0}^{q} \theta_{j} s_{j}\right)^{(m)}=\sum_{j=0}^{q} \theta_{j}^{(m)} s_{j}$ is a connection on the bundle

$$
\Delta^{m} \times P_{m} \times \Delta^{q} \rightarrow \Delta^{m} \times X_{m} \times \Delta^{q} .
$$

We have to verify that the compatibility conditions hold. The strict simplicial structure on $X_{\bullet} \times \Delta^{q}$ is given by the maps $\varepsilon_{i}^{\prime}=\varepsilon_{i} \times i d_{\Delta^{q}}$ for all $i$, where $\varepsilon_{i}$ is the map given by the strict simplicial structure on $X_{\bullet}$. We have

$$
\left(\varepsilon^{i} \times i d_{X_{m} \times \Delta^{q}}\right)^{*}\left(\sum_{j=0}^{q} \theta^{(m)} s_{j}\right)=\sum_{j=0}^{q}\left(\varepsilon^{i} \times i d_{X_{m}}\right)^{*} \theta_{j}^{(m)} s_{j}
$$

since the forms $\theta_{j}^{(m)} s_{j}$ are in

$$
\Omega_{d R}^{1}\left(\Delta^{m} \times P_{m} ; \mathfrak{g}\right) \otimes \Omega_{d R}^{0}\left(\Delta^{q}\right) \subset \Omega_{d R}^{1}\left(\Delta^{m} \times P_{m} \times \Delta^{q}\right) .
$$

Now, since the $\theta_{j}$ satisfy the compatibility conditions we have

$$
\sum_{j=0}^{q}\left(\varepsilon^{i} \times i d_{X_{m}}\right)^{*} \theta_{j}^{(m)} s_{j}=\sum_{j=0}^{q}\left(i d_{\Delta^{m-1}} \times \varepsilon_{i}\right)^{*} \theta_{j}^{(m-1)} s_{j} .
$$

As before we have

$$
\sum_{j=0}^{q}\left(i d_{\Delta^{m-1}} \times \varepsilon_{i}\right)^{*} \theta_{j}^{(m-1)} s_{j}=\left(i d_{\Delta^{m-1}} \times \varepsilon_{i}^{\prime}\right)^{*}\left(\sum_{j=0}^{q} \theta^{(m-1)} s_{j}\right),
$$

which proves the lemma

Given an invariant polynomial $\Phi$ of degree $k$, we denote by

$$
\tilde{\Theta}_{q}\left(\Phi ; \theta_{0}, \ldots, \theta_{q}\right) \in \Omega_{d R}^{2 k}\left(X_{\bullet} \times \Delta^{q}\right)
$$

the characteristic form (on $X_{\bullet}$ ) associated to $\Phi$ for the (curvature of the) connection $\sum_{j=0}^{q} \theta_{j} s_{j}$. When $\Phi$ is understood, we will omit it from the notation for the above form. The closed form $\tilde{\Theta}_{q}\left(\theta_{0}, \ldots, \theta_{q}\right)$ is a family of compatible closed forms

$$
\tilde{\Theta}_{q}^{(m)}\left(\theta_{0}, \ldots, \theta_{q}\right) \in \Omega_{d R}^{2 k}\left(\Delta^{m} \times X_{m} \times \Delta^{q}\right) .
$$

We define a form $\Theta_{q}\left(\theta_{0}, \ldots, \theta_{q}\right) \in \Omega_{d R}^{2 k-q}\left(X_{\bullet}\right)$ by

$$
\Theta_{q}\left(\theta_{0}, \ldots, \theta_{q}\right)=\int_{\Delta^{q}} \tilde{\Theta}_{q}\left(\theta_{0}, \ldots, \theta_{q}\right),
$$


i.e. $\Theta_{q}\left(\theta_{0}, \ldots, \theta_{q}\right)$ is the family of forms

$$
\Theta_{q}^{(m)}\left(\theta_{0}, \ldots, \theta_{q}\right)=\int_{\Delta^{q}} \tilde{\Theta}_{q}^{(m)}\left(\theta_{0}, \ldots, \theta_{q}\right) .
$$

These forms satisfy the compatibility conditions since the diagram

$$
\begin{aligned}
& \Omega_{d R}^{*}\left(\Delta^{m} \times X_{m} \times \Delta^{q}\right) \stackrel{\int_{\Delta^{q}}}{\longrightarrow} \Omega_{d R}^{*}\left(\Delta^{m} \times X_{m}\right) \\
& \left(\varepsilon^{i} \times \operatorname{id}_{\mathrm{X}_{\mathrm{m}} \times \Delta \mathrm{q}}\right)^{*} \downarrow \quad \downarrow\left(\varepsilon^{i} \times \mathrm{id}_{\mathrm{X}_{\mathrm{m}}}\right)^{*} \\
& \Omega_{d R}^{*}\left(\Delta^{m-1} \times X_{m} \times \Delta^{q}\right) \stackrel{\int_{\Delta^{q}}}{\longrightarrow} \Omega_{d R}^{*}\left(\Delta^{m-1} \times X_{m}\right) \\
& \left(i d_{\Delta^{m-1}} \times \varepsilon_{i}^{\prime}\right)^{*} \uparrow \quad \uparrow\left(i d_{\Delta^{m-1}} \times \varepsilon_{i}\right)^{*} \\
& \Omega_{d R}^{*}\left(\Delta^{m-1} \times X_{m-1} \times \Delta^{q}\right) \stackrel{\int_{\Delta^{q}}}{\longrightarrow} \Omega_{d R}^{*}\left(\Delta^{m-1} \times X_{m-1}\right)
\end{aligned}
$$

commutes and the forms $\tilde{\Theta}_{q}^{(m)}\left(\theta_{0}, \ldots, \theta_{q}\right) \in \Omega_{d R}^{2 k}\left(\Delta^{m} \times X_{m} \times \Delta^{q}\right)$ are compatible.

If we denote by $t$ the variables on the simplices $\Delta^{p}$, by $x$ the variables on the manifolds $X_{p}$ and by $s$ the variables on the simplex $\Delta^{q}$, then we can write with an obvious notation the differential on the complex $\Omega_{d R}^{*}\left(\Delta^{q} \times X_{\bullet}\right)$ as $\mathrm{d}=\mathrm{d}_{s}+\mathrm{d}_{t, x}$, where $\mathrm{d}_{t, x}$ is the differential of the complex $\Omega_{d R}^{*}\left(X_{\bullet}\right)$. Since $\tilde{\Theta}_{q}\left(\theta_{0}, \ldots, \theta_{q}\right)$ is closed, we have

$$
\mathrm{d}_{t, x} \tilde{\Theta}_{q}\left(\theta_{0}, \ldots, \theta_{q}\right)=-\mathrm{d}_{s} \tilde{\Theta}_{q}\left(\theta_{0}, \ldots, \theta_{q}\right) .
$$

Then we have

$$
\begin{gathered}
\mathrm{d}_{t, x} \Theta_{q}\left(\theta_{0}, \ldots, \theta_{q}\right)=\mathrm{d}_{t, x} \int_{\Delta^{q}} \tilde{\Theta}_{q}\left(\theta_{0}, \ldots, \theta_{q}\right)= \\
=\int_{\Delta^{q}} \mathrm{~d}_{t, x} \tilde{\Theta}_{q}\left(\theta_{0}, \ldots, \theta_{q}\right)=-\int_{\Delta^{q}} \mathrm{~d}_{s} \tilde{\Theta}_{q}\left(\theta_{0}, \ldots, \theta_{q}\right) .
\end{gathered}
$$

By Stokes theorem the last integral is equal to $-\int_{\partial \Delta_{q}} \tilde{\Theta}_{q}\left(\theta_{0}, \ldots, \theta_{q}\right)$, so we have proven the analogue of Theorem 3.3 in [K2].

Proposition 3.2. In the complex $\Omega_{d R}^{*}\left(X_{\bullet}\right)$ we have

$$
\mathrm{d} \Theta_{q}\left(\theta_{0}, \ldots, \theta_{q}\right)=-\sum_{i=0}^{q}(-1)^{i} \Theta_{q-1}\left(\theta_{0}, \ldots, \hat{\theta}_{i}, \ldots \theta_{q}\right) .
$$

In particular, for $q=1$ we have that given any two connections on $P_{\bullet}, \theta_{0}$ and $\theta_{1}$, and an invariant polynomial $\Phi$, we can write in a canonical way

$$
\Phi\left(\theta_{1}\right)-\Phi\left(\theta_{0}\right)=\mathrm{d} \Theta_{1}\left(\Phi ; \theta_{0}, \theta_{1}\right) .
$$

In the sequel it will be convenient to consider formal series of invariant polynomials (like the total Chern class for example), which will then give under the Chern-Weil construction formal sums of differential forms. We now describe a notation (the same as in [K2],[K3]) to write formulae in this setting in a compact way. We can write a formal series of invariant 
polynomials $\Phi$ as a sum $\sum_{r} \Phi_{r}$ with $\Phi_{r}$ a homogeneous polynomial of degree $r$. Let $\mathcal{F}=\left\{F^{r} \Omega_{d R}^{*}\left(X_{\bullet}\right)\right\}$ be a filtration of the de Rham complex of $X_{\bullet}$ and

$$
\omega=\sum_{r} \omega_{r}, \eta=\sum_{r} \eta_{r}
$$

be formal sums of forms in $\Omega_{d R}^{*}\left(X_{\bullet}\right)$ (note that we do not require that $\omega_{r}$ is of degree $r$, actually most of the times this will not be the case). We will write $\omega=\eta \bmod \mathcal{F}$ if and only if for each $r$ we have

$$
\omega_{r}-\eta_{r} \in F^{r} \Omega_{d R}^{*}\left(X_{\bullet}\right) .
$$

We will also write $\omega=\eta \bmod \tilde{\mathcal{F}}$ when for each $r$ the above equation is satisfied modulo exact forms.

With this notation, all the constructions and proofs in the sequel will be formally the same both for the case of an invariant polynomial, where we will be dealing with forms, and for a formal series of invariant polynomials, in which case we will work with formal sums of forms homogeneous degree by homogeneous degree. Hence we will not distinugish between the two cases in what follows, writing just $\Phi$ and $\omega$ also for formal sums.

Definition 3.3. Let $\Phi$ be an invariant polynomial (or a formal series) and $\mathcal{F}=\left\{F^{r} \Omega_{d R}^{*}\left(X_{\bullet}\right)\right\}$ a filtration of the de Rham complex of $X_{\bullet}$. An $(\mathcal{F}, \Phi)$ multiplicative bundle (or just a multiplicative bundle when $\mathcal{F}$ and $\Phi$ are understood) over $X_{\bullet}$ is a triple $\left(P_{\bullet}, \theta, \omega\right)$ where $P_{\bullet}$ is a principal $G$-bundle over $X_{\bullet}, \theta$ is a connection on $P_{\bullet}$ and $\omega$ is a (formal series of) form(s) in $\Omega_{d R}^{*}\left(X_{\bullet}\right)$ such that

$$
\Phi(\theta)=\mathrm{d} \omega \bmod \mathcal{F} .
$$

An isomorphism $f:\left(P_{\bullet}, \theta, \omega\right) \rightarrow\left(P_{\bullet}^{\prime}, \theta^{\prime}, \omega^{\prime}\right)$ between two multiplicative bundles is an isomorphism $f$ of the underlying bundles $P_{\bullet}, P_{\bullet}^{\prime}$ such that

$$
\omega^{\prime}-\omega=\Theta_{1}\left(\theta, f^{*} \theta^{\prime}\right) \bmod \tilde{\mathcal{F}} .
$$

As is [K2], using 3.2 to prove transitivity, it follows that isomorphism is an equivalence relation on multiplicative bundles, so we can make the following definition.

Definition 3.4. We denote by $M K^{\Phi}\left(X_{\bullet} ; \mathcal{F}\right)$ the set of isomorphism classes of multiplicative bundles, the multiplicative K-theory of $X \bullet$ with respect to $(\mathcal{F}, \Phi)$.

As usual we will omit $\Phi$ and $\mathcal{F}$ from the notation when there is no risk of ambiguity.

\section{Characteristic Classes for SeCONDARy theories}

Let $G$ be a Lie group. Given a principal $G$-bundle on a simplicial smooth manifold $X \bullet$ with a connection $\theta$, and an invariant polynomial $\Phi$ of homogeneous degree $k$ (for the case of a formal series of invariant polynomials one has just to work degree by degree as in section 3 ), we will associate 
characteristic classes with values in multiplicative cohomology groups and in groups of differential characters of $X_{\bullet}$ associated to any filtration of the simplicial de Rham complex $\Omega_{d R}^{*}\left(X_{\bullet}\right)$. This will generalize the secondary characteristic classes introduced by Karoubi in the case of smooth manifolds $[\mathrm{K} 2]$.

Let $X_{\bullet}$ be a simplicial smooth manifold, $\mathcal{F}=\left\{F^{r} \Omega_{d R}^{*}\left(X_{\bullet}\right)\right\}$ a filtration of the de Rham complex, $G$ a Lie group and $\Gamma=(P \bullet, \theta, \eta)$ a $(\mathcal{F}, \Phi)$ multiplicative bundle.

The connection $\theta$ on the principal $G$-bundle $\pi_{\bullet}$ is given as 1 -form

$$
\theta \in \Omega_{d R}^{1}\left(P_{\bullet} ; \mathfrak{g}\right)
$$

as in section 1 . The characteristic form of Theorem 1.1

$$
\Phi(\theta) \in \Omega_{d R}^{2 k}\left(X_{\bullet}\right)
$$

can also as usual be seen as a family of forms

$$
\Phi\left(\theta^{(n)}\right) \in \Omega_{d R}^{2 k}\left(\Delta^{n} \times X_{n} ; \mathfrak{g}\right)
$$

satisfying the compatibility conditions.

Since $\Gamma$ is a multiplicative bundle we have

$$
\Phi(\theta)=d \eta+\omega,
$$

where the forms $\eta$ and $\omega$ are also compatible sequences $\eta=\left\{\eta^{(n)}\right\}$ and $\omega=\left\{\omega^{(n)}\right\}$ of differential forms with $\omega \in F^{r} \Omega_{d R}^{2 k}\left(X_{\bullet}\right)$ and $\eta \in \Omega_{d R}^{2 k-1}\left(X_{\bullet}\right)$.

The connection $\theta$ is the pullback of a connection $\theta_{U_{\bullet}}$ on $U_{\bullet \bullet}$ by a map $\Psi$ as in Theorem 1.2. Let $\Lambda$ be again a subring of the complex numbers $\mathbb{C}$, and assume that $\Phi$ corresponds under the Chern-Weil map to a $\Lambda$-valued cohomology class.

For every $n$ the inclusion $\imath_{n}: B_{n} \bullet \rightarrow B_{\bullet}$ induces isomorphisms in cohomology since $\left\|B_{n} \bullet\right\|$ is homotopy equivalent to the classifying space of $G$. For every $n$ we also have that $\imath_{n}^{*} \theta_{U_{\bullet \bullet}}=\theta_{U_{n}}$. Since the form $\Phi\left(\theta_{U_{n}}\right)$ represents the class of $\Phi$ by Theorem 1.1, and $\imath_{n}^{*} \Phi\left(\theta_{U \bullet \bullet}\right)=\Phi\left(\theta_{U_{n} \bullet}\right)$, we have that the form $\Phi\left(\theta_{U \bullet \bullet}\right) \in \Omega_{d R}^{*}\left(B_{\bullet \bullet}\right)$ represents the class of $\Phi$. Then it follows that there exist a compatible cocycle $c \in C^{2 k}\left(B_{\bullet \bullet} ; \Lambda\right)$ and a compatible cochain $v \in C^{2 k-1}\left(B_{\bullet \bullet} ; \mathbb{C}\right)$ such that we have

$$
\delta v=c-\Phi\left(\theta_{U \bullet \bullet}\right),
$$

(where for simplicity we omit from the notation the maps from $\Lambda$-cochains to complex cochains and the quasi-isomorphism with the de Rham complex).

Since $\Psi^{*}$ maps compatible cochains (in the bisimplical sense) to compatible chains (in the simplicial sense), the triple $\xi(\Gamma)=\left(\Psi^{*}(c), \omega, \Psi^{*}(v)+\eta\right)$ defines a cocycle in the cone complex

$$
\operatorname{cone}\left(C^{*}\left(X_{\bullet} ; \Lambda\right) \oplus F^{r} \Omega_{d R}^{*}\left(X_{\bullet}\right) \rightarrow C^{*}\left(X_{\bullet} ; \mathbb{C}\right)\right)
$$

and since $\omega$ is a form of degree $2 k$ also a cocycle in the cone complex

$$
\operatorname{cone}\left(C^{*}\left(X_{\bullet} ; \Lambda\right) \oplus \sigma_{\geq 2 k} F^{r} \Omega_{d R}^{*}\left(X_{\bullet}\right) \rightarrow C^{*}\left(X_{\bullet} ; \mathbb{C}\right)\right) \text {. }
$$


The triple $\xi(\Gamma)$ is a cocycle, because we have $\delta \Psi^{*} c=\Psi^{*} \delta c=0$ since $c$ is a cocycle, by (4.1) we have $\mathrm{d} \omega=\mathrm{d} \Phi(\theta)+\mathrm{d}^{2} \eta=0$, and also

$$
\delta \Psi^{*} v=\Psi^{*} \delta v=\Psi^{*}\left(c-\Phi\left(\theta_{U_{\bullet}}\right)\right)=\Psi^{*} c-\Phi(\theta)=\Psi^{*} c-(\omega+\mathrm{d} \eta)
$$

The class of $\xi(\Gamma)$ is independent of the choices of $c$ and $v$ : If $c^{\prime}$ and $v^{\prime}$ are other choices satisfying (4.2) then we must have $c-c^{\prime}=\delta u$ and $\delta u=\delta\left(v-v^{\prime}\right)$. Then, since $H^{2 k-1}\left(\left\|B_{\bullet}.\right\| ; \mathbb{C}\right)$ is trivial, there exists a compatible cochain $w$ such that $\delta w=u+\left(v-v^{\prime}\right)$. If $\xi^{\prime}(\Gamma)$ is the cocycle obtained from the different choice, then $\xi(\Gamma)-\xi^{\prime}(\Gamma)=\left(\Psi^{*} \delta u, 0, \Psi^{*}\left(v-v^{\prime}\right)\right)=\mathrm{d}\left(\Psi^{*} u, 0, \Psi^{*} w\right)$.

Hence for $2 r-m=2 k$ we can define the class of the multiplicative bundle $\left(P_{\bullet}, \theta, \eta\right)$ in the multiplicative cohomology group $M H_{m}^{2 r}\left(X_{\bullet}, \Lambda, \mathcal{F}\right)$ to be the class of $\xi(\Gamma)$. Similarly the class of $\left(P_{\bullet}, \theta, \eta\right)$ in $\hat{H}_{r}^{2 k-1}\left(X_{\bullet} ; \mathbb{C} / \Lambda ; \mathcal{F}\right)$ is the class of the triple $\xi(\Gamma)$.

Proposition 4.1. The classes constructed above are characteristic classes of elements of $M K^{\Phi}\left(X_{\bullet} ; \mathcal{F}\right)$.

Proof. The naturality follows from the construction. We show that for two isomorphic multiplicative bundles $\Gamma=\left(P_{\bullet}, \theta, \eta\right)$ and $\Gamma^{\prime}=\left(P_{\bullet}^{\prime}, \theta^{\prime}, \eta^{\prime}\right)$ the cocycles $\xi(\Gamma)$ and $\xi\left(\Gamma^{\prime}\right)$ are cohomologous. We can assume $P_{\bullet}=P_{\bullet}^{\prime}$, and write $\Phi(\theta)=\omega+\mathrm{d} \eta$ and $\Phi\left(\theta^{\prime}\right)=\omega^{\prime}+\mathrm{d} \eta^{\prime}$ with $\omega, \omega^{\prime} \in F^{r} \Omega_{d R}^{2 k}\left(X_{\bullet}\right)$. Since the two multiplicative bundles are isomorphic we have

$$
\eta^{\prime}-\eta=\Theta_{1}\left(\Phi ; \theta, \theta^{\prime}\right)+\sigma+\mathrm{d} \rho
$$

with $\sigma \in F^{r} \Omega_{d R}^{2 k-1}\left(X_{\bullet}\right)$. It follows that

$$
\omega^{\prime}-\omega=\mathrm{d}\left(\Theta_{1}\left(\Phi ; \theta, \theta^{\prime}\right)-\left(\eta^{\prime}-\eta\right)\right)=-\mathrm{d}(\sigma+\mathrm{d} \rho) .
$$

Let $\Psi^{\prime}$ be the map pulling back $\Gamma^{\prime}$ given by Theorem 1.2 , let $c^{\prime}, v^{\prime}$ be the cochains used in the construction for the characteristic cycle $\xi\left(\Gamma^{\prime}\right)$. Then

$$
\xi\left(\Gamma^{\prime}\right)-\xi(\Gamma)=\left(\Psi^{\prime *} c^{\prime}-\Psi^{*} c, \omega^{\prime}-\omega, \Psi^{\prime *} v^{\prime}-\Psi^{*} v+\Theta_{1}\left(\Phi ; \theta, \theta^{\prime}\right)+\sigma+\mathrm{d} \rho\right)
$$

is cohomologous to the triple $\zeta=\left(\Psi^{\prime *} c^{\prime}-\Psi^{*} c, 0, \Psi^{\prime *} v^{\prime}-\Psi^{*} v+\Theta_{1}\left(\Phi ; \theta, \theta^{\prime}\right)\right)$ since the two differ by the coboundary of $(0,-\sigma, \rho)$. We can choose $c^{\prime}=c$ and $v^{\prime}=v+\Theta_{1}\left(\Phi ; \theta_{U \bullet \bullet}, \theta_{U \bullet \bullet}^{\prime}\right)$ (where $\theta_{U \bullet \bullet}^{\prime}$ is the connection pulling back to $\theta^{\prime}$ under $\Psi^{\prime *}$ given by theorem 1.2) satisfying (4.2), hence we have, using also the naturality of the first transgression form,

$$
\zeta=\left(\Psi^{\prime *} c-\Psi^{*} c, 0, \Psi^{\prime *} v-\Psi^{*} v+\Theta_{1}\left(\Phi ; \Psi^{\prime *} \theta_{U \bullet \bullet}, \theta^{\prime}\right)+\Theta_{1}\left(\Phi ; \theta, \theta^{\prime}\right)\right) .
$$

Using Proposition 3.2 , we have that

$$
\begin{array}{r}
\Theta_{1}\left(\Phi ; \Psi^{*} \theta_{U \bullet \bullet}, \Psi^{\prime *} \theta_{U \bullet \bullet}\right)+\mathrm{d} \Theta_{2}\left(\Phi ; \Psi^{*} \theta_{U \bullet \bullet}, \Psi^{\prime *} \theta_{U \bullet \bullet}, \theta^{\prime}\right)= \\
\Theta_{1}\left(\Phi ; \Psi^{\prime *} \theta_{U \bullet \bullet}, \theta^{\prime}\right)+\Theta_{1}\left(\Phi ; \Psi^{*} \theta_{U \bullet \bullet}, \theta^{\prime}\right) .
\end{array}
$$

Since $\Psi^{\prime}$ and $\Psi$ are homotopic, there is a chain homotopy $H$ between the induced cochain maps; using $H$ we can write $\zeta$ as

$$
\left(\delta H c, 0, \delta H v+H \delta v+\Theta_{1}\left(\Phi ; \Psi^{*} \theta_{U \bullet \bullet}, \Psi^{\prime *} \theta_{U \bullet \bullet}\right)+\operatorname{d} \Theta_{2}\left(\Phi ; \Psi^{*} \theta_{U \bullet \bullet}, \Psi^{\prime *} \theta_{U \bullet \bullet}, \theta^{\prime}\right)\right) .
$$


Then $\zeta$ is cohomologous to $\left(\delta H c, 0,+H \delta v+\Theta_{1}\left(\Phi ; \Psi^{*} \theta_{U \bullet \bullet}, \Psi^{\prime *} \theta_{U \bullet \bullet}\right)\right)$, and since the transgression forms $\Theta_{1}(\cdot ; \cdot, \cdot)$ are compatible with chain homotopies (see [DHZ], appendix A), the former cocycle is cohomologous to

$$
\left(\delta H c, 0, H \delta v+H \Phi\left(\theta_{U \bullet \bullet}\right)\right)=\mathrm{d}(H c, 0,0)
$$

because $H c=H\left(\delta v+\Phi\left(\theta_{U \bullet \bullet}\right)\right)$ by (4.2).

Remark 4.2. The above characteristic classes can be slightly generalized: suppose $\Phi$ and $\Phi^{\prime}$ are formal sums of invariant polynomials such that every $(\mathcal{F}, \Phi)$-multiplicative bundle is also a $\left(\mathcal{F}, \Phi^{\prime}\right)$-multiplicative bundle (the main example we have in mind is the Chern character $c h$ and the total Chern class c). Then by the same procedure we can construct the classes associated to $\Phi^{\prime}$ of elements of $M K^{\Phi}\left(X_{\bullet} ; \mathcal{F}\right)$ with values in the multiplicative cohomology groups and in the groups of differential characters associated to $\mathcal{F}$.

\section{REFERENCES}

[B] K. Behrend, On the de Rham Cohomology of Differential and Algebraic Stacks, preprint arXiv:math.AG/0410255

[CS] J. Cheeger, J. Simons, Differential characters and geometric invariants. Geometry and topology (College Park, Md., 1983/84), 50-80, Lecture Notes in Mathematics, Vol. 1167, Springer-Verlag, Berlin-New York, 1985.

[CM] M. Crainic, I. Moerdijk, Čech-de Rham theory for leaf spaces of foliations. Math. Ann. 328 (2004), no. 1-2, 59-85.

[De] P. Deligne, Théorie de Hodge II, Publ. Math. IHES 40 (1971), 5-57.

[D1] J. Dupont, Simplicial de Rham cohomology and characteristic classes of flat bundles, Topology 15 (1976), no. 3, 233-245.

[D2] J. Dupont, Curvature and characteristic classes, Lecture Notes in Mathematics, Vol. 640 Springer-Verlag, Berlin-New York, 1978.

[DHZ] J. Dupont, R. Hain, S. Zucker, Regulators and characteristic classes of flat bundles. The arithmetic and geometry of algebraic cycles (Banff, AB, 1998), 47-92, CRM Proc. Lecture Notes, 24, Amer. Math. Soc., Providence, RI, 2000.

[DJ] J. Dupont, H. Just, Simplicial currents, Illinois Journ. of Math., Vol. 41 (1997), No. $1,354-377$.

[E1] H. Esnault, Characteristic classes of flat bundles. Topology 27 (1988), no. 3, 323-352.

[E2] H. Esnault, Algebraic differential characters. Regulators in analysis, geometry and number theory, 89-115, Progr. Math., 171, Birkhuser Boston, 2000.

[F] M. Felisatti, Differential characters and multiplicative cohomology, K-Theory 18 (1999), no. 3, 267-276.

[G] E. Getzler, The equivariant Chern character for non-compact Lie groups, Adv. Math. 109 (1994), no. 1, 88-107.

[HS] M. J. Hopkins, I. M. Singer, Quadratic functions in geometry, topology, and Mtheory, preprint arXiv:math.AT/0211216

[K1] M. Karoubi, Homologie cyclique et $K$-théorie, Astérisque No. 149 (1987).

[K2] M. Karoubi, Théorie générale des classes caractéristiques secondaires, $K$-Theory 4 (1990), no. 1, 55-87.

[K3] M. Karoubi, Classes caractéristiques de fibrés feuilletés, holomorphes ou algébriques. K-Theory 8 (1994) no. 2, 153-211.

[LTX] C. Laurent-Gengoux, J.-L. Tu, P. Xu, Chern-Weil map for principal bundles over groupoids, preprint arXiv:math.DG/0401420. 
[LU] E. Lupercio, B. Uribe, Differential characters for orbifolds and string connections I, to appear: Proceedings of "Gromov-Witten theory of spin curves and orbifolds, AMS meeting San Francisco 2003", preprint arXiv:math.DG/0311008.

[S] G. Segal, Classifying spaces and spectral sequences, Inst. Hautes Études Sci. Publ. Math. No. 34 (1968), 105-112.

Department of Mathematics, University of Leicester, University Road, LeICESTER LE1 7RH, ENGLAND, UK

E-mail address: mf46@mcs.le.ac.uk

Department of Mathematics, University of Leicester, University Road, LeICESTER LE1 7RH, ENGLAND, UK

E-mail address: fn8@mcs.le.ac.uk 